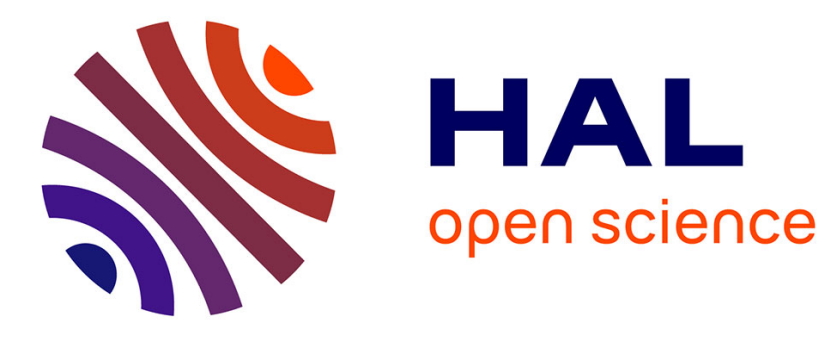

\title{
L'École et la crise des "configurations d'assujettissement"
}

Jean-François Nordmann

\section{To cite this version:}

Jean-François Nordmann. L'École et la crise des "configurations d'assujettissement". Le Télémaque. Philosophie, Education, Société, 2013, 43 (1), pp.59-71. 10.3917/tele.043.0059 . hal-03569063

\section{HAL Id: hal-03569063 https://hal.science/hal-03569063}

Submitted on 12 Feb 2022

HAL is a multi-disciplinary open access archive for the deposit and dissemination of scientific research documents, whether they are published or not. The documents may come from teaching and research institutions in France or abroad, or from public or private research centers.
L'archive ouverte pluridisciplinaire HAL, est destinée au dépôt et à la diffusion de documents scientifiques de niveau recherche, publiés ou non, émanant des établissements d'enseignement et de recherche français ou étrangers, des laboratoires publics ou privés. 


\section{L'École et la crise des « configurations d'assujettissement » \\ Jean-François Nordmann \\ Référence : \\ Nordmann, J.-F. (2013). L'École et la crise des \\ « configurations d'assujettissement ». Le Télémaque, 43, 59-71.}

On avancera dans les pages qui suivent une thèse - ou du moins une hypothèse - qui peut sans doute paraître singulière et paradoxale : le projet de l' « émancipation », qui semble inscrit au cœur même de notre horizon éducatif et politique depuis la philosophie des Lumières, serait en fait un projet d' « assujettissement» et en l'occurrence de constitution des sujets par inhibitionrépression et assignation à des Lois supérieures. Les institutions explicitement porteuses de ce projet, autrement dit notre École et notre République - mais aussi peut-être, au-delà, nos autres institutions et modes d'organisation sociale et sociétale - pourraient dans cette perspective être tenues pour autant de «configurations d'assujettissement », travaillant de façon coordonnée ou convergente à la production de ce processus de l' « assujettissement».

Cependant, l'idée complémentaire qu'on soutiendra est que nous nous trouverions aujourd'hui à un moment historique décisif où ces « configurations d'assujettissement $»$, tout en continuant de fonctionner et de conserver une emprise considérable, seraient entrées dans une crise et une mise en question profondes venant notamment produire un ébranlement sans précédent de la représentation de l'École, de la République et de l'horizon même de l'émancipation par l'éducation et la politique. L'issue même de cette crise resterait profondément incertaine, pouvant conduire à des évolutions très différentes qu'il importe de chercher à appréhender et à préciser.

\section{Émancipation, instruction et accès à la raison}

Si l'on considère pour commencer l'idée même d' " émancipation », on relèvera que, dans l'acception qui nous intéresse ici (et qui fait qu'on parle d'École ou de République «émancipatrice »), l'émancipation renvoie toujours à l'idée d'un processus de libération ou d'affranchissement — de passage d'un état initial de sujétion, de servitude, de privation de liberté, à un état final de jouissance de liberté - qui ne concerne pas seulement un seul individu ou quelques-uns (comme dans l'acte d'émancipation d'un esclave ou d'un mineur), mais qui concerne fondamentalement chaque individu en même temps que tous les autres et conjointement avec eux. L'émancipation, autrement dit, apparaît comme un processus qui est toujours indissociablement individuel et collectif : la libération de chacun est celle de tous ensemble et de tous également. Elle est par ailleurs toujours dans l'acception qui nous intéresse ici — usuellement chargée de connotations absolutisantes voire eschatologiques propices à son instauration en horizon ultime de pensée et d'action : elle est ce qui permettrait à la fois de faire accéder définitivement les individus à la liberté et d'inaugurer le règne de "la" bonne politique - celle qui instaurerait véritablement le régime de la liberté et de l'égalité de tous les hommes.

Mais comment se représente-t-on usuellement l'action ou le pouvoir « émancipateur» de l'École ? Il semble qu'on associe immédiatement le processus d'émancipation à la mise en œuvre de l'« instruction» et par suite (pour les sujets 
élèves) de à l'accès même au savoir, mais à un accès au savoir de type tout à fait particulier. Précisons les choses. L'émancipation implique la représentation d'un état initial ou originaire de sujétion dans lequel se trouverait pris le sujet, sous l'emprise de son environnement immédiat (avec son caractère borné et limité et ses contraintes objectives), mais aussi des multiples pouvoirs extérieurs ou « tutelles » (parentales, religieuses, idéologiques...) qui travaillent à diriger ou à infléchir sa pensée et sa conduite, et enfin - emprise d'autant plus redoutable qu'elle est inaperçue — de ses propres opinions et préjugés. L'arrachement à cet état de foncière sujétion initiale requerrait dès lors bien plus que le simple accès à des savoirs pratiques utiles ou à des savoirs simplement factuels et extérieurs : il demanderait non seulement que les sujets développent réellement, fût-ce à un niveau "élémentaire ", l'ensemble des savoirs - y compris théoriques et scientifiques — du monde et des hommes, mais qu'ils produisent réellement au moins en partie ces savoirs par eux-mêmes, autrement dit qu'ils fassent réellement des mathématiques, de la physique, de l'histoire, de la philosophie..., bien au-delà d'un savoir qui ne porterait que «sur » ces disciplines et leurs résultats, leurs possibles applications, etc. Et cela parce que l'enjeu de cette production même du savoir serait, au travers de la mise en cuvre des capacités de réflexion, de raisonnement, d'argumentation, de prise en compte des objections, de conscience critique, etc., le développement en eux d'une "raison » leur permettant peu à peu de se constituer en sujets capables d'analyser, de juger et de penser par eux-mêmes tout ce qui peut leur arriver - et par suite aussi d'agir et de conduire leur vie par eux-mêmes. L'émancipation consisterait ainsi dans cette formation ou cette production de sujets rationnels et "autonomes », auxquels il n'y aurait par exemple plus lieu, sur le plan éducatif, d'inculquer de bonnes valeurs préétablies, mais seulement de donner tous les éléments pour qu'ils puissent décider par eux-mêmes des bonnes valeurs - avec la présupposition que de tels sujets, en tant que rationnels, ne peuvent aussi être que des sujets qui se soucient du bien des autres sur le plan moral comme du bien commun sur le plan politique.

Mais c'est précisément cette opération fondamentale de constitution des sujets en sujets rationnels, autonomes, moraux et citoyens, qui nous apparaît pouvoir être comprise comme un processus d'«assujettissement» et qui incite à concevoir l'ensemble de l'École émancipatrice comme «configuration d'assujettissement». Mais il faut préciser en quel sens précis on propose ici de parler d' « assujettissement » et de « configuration d'assujettissement ».

\section{« Assujettissement » et « configuration d'assujettissement »}

On prendra ici le terme d' ' assujettissement» en un sens spécifique, distinct du sens général qu'il prend quand, selon son acception ordinaire, il désigne l'opération par laquelle les sujets se trouvent objectivement contraints par la force à l'obéissance et la soumission absolues à un pouvoir extérieur, politique ou social. L'« assujettissement» que nous avons ici en vue n'est pas un tel processus d'« asservissement» ou d'« inféodation ». Il n'en désigne pas moins un processus qui s'accompagne de contrainte intérieure, et même de contrainte intérieure profonde, même si le sujet peut ne ressentir cette contrainte que de façon très confuse, sous la forme d'un mal-être diffus, inexplicitable et inexplicable.

Ce processus d'assujettissement, nous le définirons comme ce processus par lequel un sujet se trouve constitué au plus profond de lui-même par son (auto)assignation à une Loi qui se donne à lui, à la fois et dans le même temps, comme un commandement impérieux supérieur auquel il ne peut se soustraire et comme un principe essentiel ou fondateur qui soutient toute son existence et son être. Deux traits phénoménologiques à première vue antithétiques sont ainsi étroitement 
associés et conjugués dans ce rapport spécifique à la Loi que met en jeu l'assujettissement : d'une part la Loi s'impose comme depuis une position de surplomb ou de transcendance et de façon impérative catégorique ; d'autre part, elle est immédiatement perçue et éprouvée comme une condition fondamentale de possibilité sans laquelle ni la vie individuelle ni la vie collective (la coexistence des sujets, leur vie sociale, leur être ensemble) ne seraient de fait garanties de pouvoir subsister et sans laquelle par ailleurs ni cette vie individuelle ni cette vie collective ne pourraient non plus être élevées au-dessus du «simple » niveau d'une existence " immédiate» pour atteindre au plan "supérieur» de quelque "essence» et « destination » de l'Humanité par exemple en tant qu'Esprit, ou Raison, ou Progrès. Une telle Loi, qui peut se décliner en diverses figures - Loi divine, Loi naturelle, Loi morale, Loi rationnelle, Loi civilisationnelle... - force donc une obéissance sans appel tout en s'accompagnant de la reconnaissance de sa nécessité de fait et de sa valeur de fondement essentiel. Mais la soumission qu'elle impose n'est pas alors incompatible avec la liberté ou du moins avec une certaine forme de liberté : bien plus, elle la requiert même, puisqu'il s'agit bien pour le sujet, en même temps qu'il s'éprouve contraint par elle, de reconnaître expressément par lui-même, en son for intérieur, la valeur de cette Loi qu'il se trouve dès lors également disposé non pas seulement à accepter mais à désirer et à vouloir activement. Mais c'est là la forme même de liberté que l'on désigne dans la tradition philosophique - et conformément à l'étymologie — comme " autonomie », selon un sens spécifique (d'auto-donation de Loi ou ici d'auto-assignation à une Loi qu'on découvre au fond de soi) qu'il faut évidemment distinguer de l'acception générale et commune qui assimile (comme plus haut) l'« autonomie » à la simple autarcie et absence de dépendance par rapport aux autres. Il n'y a donc pas ainsi finalement contradiction entre « assujettissement» et liberté : le sujet doit être libre pour pouvoir s'auto-assigner à la Loi, et cette autoassignation lui permet en retour de s'affranchir du poids de son environnement, de ses tutelles extérieures comme de ses opinions et préjugés pour ne plus relever et dépendre que de lui-même. On voit ainsi également comment, loin d'être contradictoires, «émancipation» et «assujettissement» peuvent venir à se conjoindre et à apparaître même indissociables - notre thèse ou notre hypothèse étant que c'est un tel «assujettissement» qui est au cœur de ce que l'École et la République entendent instaurer au titre de l' "émancipation» et de l'accès à la raison, à la morale et à la citoyenneté.

Mais avant de développer ce point, on précisera que le processus d'assujettissement tel que nous l'entendons ici consiste bien en un véritable processus de production et de structuration d'un sujet et de l'ensemble de son rapport au monde, autrement dit en un véritable processus de subjectivation, au terme duquel le sujet se trouve disposé à ne pas pouvoir se concevoir ni concevoir son rapport aux autres en dehors de cette instance de Loi et de ce rapport à la Loi que nous avons essayé de caractériser ; la forme de son monde et ses horizons de sens et d'existence se trouvent ainsi prédéterminés, et cela sans que cette prédétermination soit appréhendée et éprouvée comme telle. C'est ce qui nous fait introduire ici la notion de «configurations d'assujettissement», pour désigner par là les ensembles structurés et convergents de pratiques et de représentations collectives qui produisent et soutiennent sans cesse ce processus singulier de subjectivation par (auto)assignation à une Loi transcendante et constituante. On pourra ainsi parler de «l'École» et de «la République» comme de configurations d'assujettissement, qu'on les regarde comme deux configurations d'assujettissement distinctes conjuguant et renforçant leurs actions respectives, ou comme deux manifestations ou déclinaisons particulières d'une même configuration globale d'assujettissement. 
On relèvera - et c'est un point décisif - qu'une configuration d'assujettissement ainsi entendue ne doit pas être confondue avec un régime politique ou social de domination, d'oppression et d'exploitation, ni de façon plus générale avec un "dispositif de pouvoir» au sens de M. Foucault ou de P. Bourdieu. Les sujets produits par une configuration d'assujettissement ne se soumettent en effet qu'à une Loi qu'ils reconnaissent intérieurement et à laquelle ils s'auto-assignent librement. Par ailleurs, à la différence d'un dispositif de domination ou de pouvoir, qui fonctionne au profit de certains individus ou de certaines classes au détriment des autres, le processus d'assujettissement opère le plus souvent également dans tous les sujets, et dans les sujets des classes dominantes ou " privilégiées » non moins que dans les autres, ce qui signifie qu'elle se déploie à un niveau spécifique qui ne coïncide pas (même s'il lui est sans doute le plus souvent lié) avec celui de l'organisation sociale d'un régime de domination ou de "privilèges". Et c'est là ce qui justifie qu'on utilise ici le terme de "configuration»: à la différence du «dispositif» qui renvoie à l'idée d'un instrument intentionnellement produit, maîtrisable et faisant l'objet d'une mise en œuvre calculée, la «configuration » renvoie bien plutôt à l'idée d'une structuration d'ensemble qui est englobante et que personne n'a au départ conçue ni n'est ensuite en position de piloter ou de manipuler à ses propres fins.

Mais il faut aller plus loin et introduire l'idée que, loin de viser par elle-même l'asservissement ou l'inféodation des sujets, cette création collective et en grande partie inconsciente qu'est une configuration d'assujettissement vise ou viserait fondamentalement le bien même des sujets, et leur bien individuel tout autant que leur bien collectif. C'est du moins ainsi qu'elle se présente et qu'elle se justifie "de l'intérieur" : on relèvera en effet que l'(auto-)assignation à une Loi comporte toujours comme corrélat la mise en jeu d'une représentation singulière suivant laquelle il y aurait au fond de l'être humain quelque mauvais fond de désirs sauvages, brutaux, bestiaux... (qui peuvent prendre diverses figures : "désirs irrationnels », " péché » « passions », « pulsions »...) et qui ne pourraient pas être contrôlés ou contenus sinon par l'imposition d'une violence ou d'une contre-violence de force au moins égale. L'assujettissement serait dès lors fondamentalement de nature protectrice : il servirait à inhiber et réprimer ce vieux fond de sauvagerie et d'irrationalité qui est en chacun et d'où peuvent à tout moment déferler la barbarie et le chaos. Et l'on comprend qu'à ce titre, et très loin donc des dispositifs subis de pouvoir ou de domination, une configuration d'assujettissement puisse faire l'objet d'une très ample - voire unanime - adhésion collective.

\section{L'École comme configuration d'assujettissement}

Mais ne peut-on regarder notre École - celle que nous connaissons depuis la fin $\mathrm{du} \mathrm{XIX}^{\mathrm{e}}$ siècle et qui continue encore largement de fonctionner aujourd'hui comme une telle configuration d'assujettissement ? C'est ce que l'on soutiendra, en relevant ici cinq traits caractéristiques qui appellent l'attention et demandent à être mis en corrélation les uns avec les autres.

$1^{\circ}$ ) La discipline, au sens de la mise en ordre contraignante des corps et des conduites individuelles et collectives, apparaît d'abord occuper dans notre École une place considérable, et cela non pas seulement à ses premiers niveaux, mais continûment et jusqu'au terme même de la scolarité. Et le point n'est pas d'ailleurs seulement quantitatif: si l'on y regarde de près, on voit en effet qu'on n'a pas seulement recours pragmatiquement à la discipline comme à un moyen malheureusement nécessaire (mais provisoire) pour créer et garantir les conditions propices à l'apprentissage, mais qu'elle est en fait mise en œuvre - et expressément 
assumée - comme une action ayant sa fin en soi et de portée éducative fondamentale. Obéir pour obéir se justifie ainsi au départ comme exercice préparatoire à l'(auto-)soumission à l'impératif catégorique de la Loi, mais n'est pas moins requis par la suite pour des sujets qui ont certes accédé à la conscience morale et rationnelle et à la reconnaissance de la Loi, mais qui restent cependant - et à jamais - habités par ce mauvais fond irréductible de désirs auxquels ils doivent se contraindre de ne pas céder.

$2^{\circ}$ ) On relèvera combien, malgré le développement des pédagogies dites «actives» au $\mathrm{XX}^{\mathrm{e}}$ siècle, et malgré les déclarations d'intentions et parfois les instructions officielles mêmes de l'Ecole, les pédagogies dites «magistrales" continuent d'y exercer une emprise considérable, pédagogies où non seulement l'enseignant reste continûment en positionnement frontal, vertical et directif par rapport aux élèves, les tenant « sous » son regard et «sous » sa parole et ne cessant de leur donner ordres et consignes de travail et de conduite même lorsqu'il s'agit de les faire travailler "activement», mais où il escompte aussi que les élèves reconnaissent le bien-fondé et la nécessité de sa posture en surplomb et que cette expérience, constitutive de son «autorité », soit pour eux une de leurs expériences formatrices fondamentales. L'emprise des pédagogies «magistrales » ne serait donc nullement l'effet de quelque résistance inertielle du "système", mais serait au contraire le résultat d'un choix profond et délibéré - quoique restant inexplicité. Et c'est ce qui expliquerait de même la singulière limitation des termes mêmes autour desquels vient à se construire ensuite le débat traditionnel sur l'autorité de l'enseignant, débat où l'on n'entrevoit en général que deux options : la posture sévère-autoritaire (« Tu fais çà parce que c'est comme çà ») et la posture explicativelégitimante (« Tu fais çà parce c'est comme çà que tu réussiras à apprendre, à progresser... »), sans que vienne jamais à être envisagé qu'il puisse y avoir de tout autres postures pédagogiques qui ne sont pas frontales-directives.

$3^{\circ}$ ) On relèvera également comment les savoirs enseignés sont présentés aux élèves, et souvent là encore jusqu'à la fin même de leur scolarité, comme étant d'une part déjà pré-établis et pré-constitués, et comme ouvrant d'autre part à quelque plan ou ordre supérieur de la Vérité ou en tout cas de la Raison, de l'Intellectualité, du Progrès, de l'Esprit... Ce faisant, ils apparaissent bien fonctionner comme de véritables Lois transcendantes et constituantes au sens où on en a parlé plus haut. On ne s'étonnera pas dès lors - s'il est vrai que c'est cela même qui est recherché et qui est conçu comme un aspect fondamental de la formation - que les élèves éprouvent de leur côté corrélativement que ces savoirs sont profondément coupés de leurs expériences vécues, de leurs pré-savoirs, de leurs questions spontanées... qui n'ont pas à trouver leur place à l'École, et se représentent petits et écrasés face à ces savoirs qui descendent d'un lointain Ciel éthéré pour se mettre à leur portée.

$4^{\circ}$ ) L'Ecole apparaît encore mettre en œuvre un rapport fondamental au passé qui est singulier, où ce passé est représenté comme source à la fois de la disposition d'un bien légué fondamental voire fondateur et de l'assignation d'un devoir — non moins fondamental et fondateur - de préservation, de transmission et de fidélité. C'est ce rapport, avec sa double modalité de don et de dette, qui est au cœur de ce qu'on pourrait appeler processus de «patrimonialisation » du passé, de l'histoire, de la culture, de l'art... et qui semble mis en jeu chaque fois que les programmes et les instructions scolaires font état d'un accès aux "grandes figures » et "événements fondateurs » de l'histoire ou aux «grands textes», aux "grandes œuvres», aux « œuvres majeures» de la littérature, de l'art, de la philosophie... Il n'apparaît nullement fortuit par ailleurs que soit régulièrement associée à ce processus de «patrimonialisation» l'idée, explicite ou implicite, d'une communauté d'appartenance ou d'identité (diversement déclinable en tant que nationale, 
historique, culturelle, civilisationnelle, etc.) où chaque sujet aurait à reconnaître une coordonnée fondamentale de son existence à la fois en tant qu'origine et en tant que destin.

$5^{\circ}$ ) Enfin, au cœur même de l'éducation morale et civique qui occupe évidemment le centre du projet éducatif de l'Ecole, il ne serait pas difficile de relever comment l'« autonomie » à laquelle il s'agit de conduire les élèves vient toujours à être immédiatement déterminée et comprise comme « responsabilité », et comment à son tour cette " responsabilité » vient s'expliciter comme reconnaissance et autoprescription de devoirs de « respect» (envers soi-même, envers les autres, envers les règles et les valeurs du collectif...) qui seraient prescrits par des Lois transcendantes et constituantes (raison, humanité, droits de l'homme...).

\section{Le travail conjoint des configurations d'assujettissement}

Mais l'École est-elle seule à fonctionner ainsi comme configuration d'assujettissement? On pourrait à première vue l'imaginer et se représenter qu'il est peut-être nécessaire que règne un tel régime d'assignation à des Lois durant le temps de l'éducation et de la formation (fût-il prolongé jusqu'à la fin de la scolarité et l'entrée dans la majorité) pour pouvoir laisser place ensuite à un régime accompli de liberté, de libre conduite de sa vie et de libre auto-détermination. Mais ce n'est pas ce que l'on observe. F. Dubet relève ainsi, dans Le déclin de l'institution ${ }^{1}$, comment c'est tout l'ensemble du « travail sur autrui », sous toutes ses diverses formes (travail éducatif, social, médical...), qui fonctionne ainsi en venant sans cesse réassigner le sujet ignorant, exclu, malade... à des valeurs et des principes supérieurs (Raison, Bien collectif, Science, ...) ; et il fait de ce mode de fonctionnement, désigné comme « programme institutionnel », une caractéristique même de toute la modernité. Mais on pourrait se demander si, au-delà même, ce n'est pas tout l'ordre familial, social, politique - et, au-delà encore, tout l'ordre subjectif et inter-subjectif - qui est organisé selon de semblables configurations d'assujettissement. Ainsi, et sans parler même de l'éducation parentale et familiale où l'apprentissage de la discipline et de l'autorité apparaît occuper une place aussi centrale que dans l'École, le fonctionnement traditionnel paternel et patriarcal, qui conserve encore une emprise profonde aujourd'hui, apparaît reposer sur l'auto-assignation de chacun (père et mère, fils et filles) à la Loi supérieure qui lui confère, de façon contraignante mais constitutive, sa place et sa fonction dans l'accomplissement de la filiation patrilinéaire et de la transmission patronymique et patrimoniale. On peut de même constater l'ampleur considérable que garde l'organisation hiérarchisante des rapports professionnels comme des rapports sociaux de classes, avec les représentations qui lui sont associées de verticalité à la fois surplombante et constituante. De leur côté, les rapports politiques, même dans nos Républiques soi-disant démocratiques, continuent pour une grande part de s'organiser autour de la figure cardinale de l'État et de son autorité "supérieure», figure que n'entament pas les représentations pourtant bien répandues du contrat social et de la volonté générale. A d'autres niveaux enfin, il est frappant de voir que quelque chose du religieux et du besoin même du religieux semble demeurer et résister encore avec force au temps même où l'ensemble de la représentation du monde s'est foncièrement sécularisée et rationalisée, ou bien de quelle façon les rites de convivialité et de commensalité continuent de s'organiser autour du plat dit «de résistance» et de la singulière obligation qui est faite à chacun de prendre part à la perpétration collective d'une

\footnotetext{
${ }^{1}$ F. Dubet, Le déclin de l'institution, Paris, Éditions du Seuil, 2002.
} 
mise à mort et d'un meurtre sous l'injonction d'une Loi supérieure immorale et pourtant nécessaire et constituante.

\section{L'entrée en crise des configurations d'assujettissement}

En dépit de l'emprise très profonde et persistante de ces multiples configurations d'assujettissement sociales et sociétales et de leurs effets conjugués, on soutiendra pourtant l'idée que nous serions entrés depuis déjà quelques décennies dans une crise générale et systémique de toutes ces configurations d'assujettissement, se déclinant en de multiples crises particulières - crises de l'École, des institutions médicales et sociales, de l'éducation parentale, de la représentation de la filiation, des rapports professionnels et sociaux, du modèle républicain, etc. Mais comment se serait produite cette entrée en crise ? Serait-elle liée à quelque événement particulier, par exemple à l'événement «Mai 68 » dont on observe que, de façon significative, il marque profondément la conscience et la mémoire collectives et leur reste pourtant jusqu'à aujourd'hui encore partiellement obscur et énigmatique ? Ou bien adviendrait-elle comme un processus très lent et graduel et pouvant même passer largement inaperçu, par exemple à la manière d'une insensible et constante érosion se traduisant par le fait que, là où les configurations d'assujettissement avaient jusque là fait l'objet d'un consentement collectif très large voire unanime, elles continuent bien de fonctionner aujourd'hui mais en suscitant une certaine réserve et réticence diffuse dont on ne sait pas dire la raison.

Quoi qu'il en soit, cette crise dont nous faisons ici l'hypothèse s'avèrerait constituer un événement ou un processus de portée historique considérable, venant mettre en question un régime multiséculaire de l'assujettissement qui n'aurait donc pas concerné seulement la formation éducative initiale, mais tout l'ensemble de la production sociale des subjectivités. Mais, de l'intuition à la proposition construite, le chemin serait long : circonscrire historiquement ce régime multiséculaire n'est pas une tâche aisée, même si l'on peut être tenté, à titre de simple piste initiale, de l'associer à l'époque dite de la " modernité » (modernité d'abord religieuse et critique avec la Réforme, puis laïque, républicaine et "émancipatrice» à partir de la philosophie des Lumières) et de l'opposer à ce qui aurait été, en amont (mais on tombe ici dans une spéculation bien hasardeuse), un régime caractéristique des sociétés dites « traditionnelles » où les processus de subjectivation seraient encore beaucoup plus sommaires et contraignants, assignant les individus à une certaine place et fonction par l'efficace d'une prescription imposée sans la condition même de sa reconnaissance intérieure par les sujets.

Mais quelles peuvent être, au-delà des modalités de sa survenue, les raisons de cette entrée en crise des configurations d'assujettissement ? On suggèrera ici trois directions d'explication :

$1^{\circ}$ ) On avancera l'idée que les expériences historiques du $\mathrm{XX}^{\mathrm{e}}$ siècle ont joué un rôle déterminant en générant un sentiment, largement partagé, que les configurations éducatives, sociales, politiques... qui soutenaient les sociétés occidentales n'avaient pas empêché celles-ci de basculer et de plonger dans des formes et des degrés inédits de passages à l'acte meurtriers, de destructions et d'auto-destructions - ces désastres collectifs attestant donc de la fragilité et de la vulnérabilité de ces configurations et de l'échec même de cette raison, de cette morale et de cette citoyenneté républicaine censées protéger les sujets du déferlement de la sauvagerie et du chaos.

$2^{\circ}$ ) On suggèrera aussi, en allant plus loin, qu'une perception collective s'est également formée, même diffuse et inexplicitée, que les configurations 
d'assujettissement, loin d'avoir seulement échoué à les empêcher, avaient peut-être directement contribué à générer ces désastres collectifs. Et, à la réflexion, on pouvait en effet considérer d'abord que, produisant la représentation d'une communauté d'appartenance, d'origine et de destin sous l'égide d'une Loi transcendante, elles prêtaient aisément à la formation de politiques identitaires ou identitaristes agressives et mortifères. Mais on pouvait aussi se demander si l'(auto-)assignation des individus à des Lois supérieures ne générait pas toujours en eux d'intenses affects de frustration, de ressentiment et de haine, pouvant justement conduire à des passages à l'acte et particulièrement sous la forme de déchaînements de violences collectives. A un double titre donc, les configurations d'assujettissement pouvaient apparaître comme faisant partie des sources ou des causes mêmes du mal dont elles étaient censées être le remède.

$3^{\circ}$ ) Enfin, à un autre niveau, on retiendra l'idée que la mise en question de ces configurations d'assujettissement se trouve peut-être aussi liée à un processus historique beaucoup plus large qu'on désigne classiquement, après N. Elias, comme individualisation croissante des individus, et qu'on peut lier non pas à quelque marche en avant de l'Humanité ou de l'Histoire, mais à tout un faisceau de causes ou de facteurs contingents et conjoncturels tels que progrès de la médecine, accroissement de la longévité, développement d'une contraception efficace, multiplication des moyens de transport, de communication, d'accès à l'information, etc. Les manifestations de cette individualisation croissante seraient en tout cas multiples. Ainsi chacun se voit reconnaître socialement des espaces nouveaux et sans cesse croissants de liberté dans sa manière de vivre, de mener ses choix professionnels, d'orienter sa vie sexuelle, etc., tandis qu'une attention de plus en plus grande vient à être portée, par les développements de la psychologie et de la psychanalyse, aux vécus et aux affects individuels (auparavant traités comme reste négligeable du fonctionnement de l'ordre collectif) et que la politique républicaine, de son côté, poursuit (ou du moins affiche) le programme d'étendre toujours davantage la reconnaissance et la protection d'une sphère individuelle "privée » qu'il s'agirait de soustraire à la supposée ingérence de la sphère "publique ». Sans doute peut-on faire l'hypothèse que toutes ces évolutions et transformations conduisent peu à peu les sujets à vouloir cesser de vivre dans l'économie psychique de la répression et du "malaise dans la civilisation » et contribuent à affaiblir ou à rendre de plus en plus problématique la représentation de l'existence du mauvais fond irréductible d'irrationalité et de sauvagerie au cœur de l'être humain.

Ces raisons qu'on a indiquées - expérience de l'échec historique de la « civilisation » occidentale (rationnelle, morale et républicaine), compréhension des effets psychiques pathogènes des configurations d'assujettissement, processus d'individualisation croissante des individus - ne sont évidemment que de premières pistes. Mais elles permettent peut-être de contribuer à éclairer quelque peu la profondeur de cette crise qui n'atteint pas seulement l'École, mais tout l'ensemble du processus et des configurations d'assujettissement.

Perspectives sur les évolutions possibles de la crise

Cette interprétation que nous avons proposée nous laisse-t-elle seulement avec un diagnostic de crise généralisée, ou bien permet-elle d'ouvrir quelques perspectives concernant le devenir possible des configurations d'assujettissement et particulièrement de la configuration d'assujettissement de l'École ? On avancera ici, pour terminer, trois propositions :

$1^{\circ}$ ) On soutiendra d'abord que nous nous trouvons sans doute à un moment historique particulièrement dangereux, non pas parce que nous vivons cette crise 
profonde des configurations d'assujettissement, mais parce qu'elle menace très effectivement de livrer les individus, et le collectif tout entier, à l'emprise d'un très puissant individualisme hédoniste qui ne reconnaîtrait plus comme horizon de vie que la satisfaction immédiate des besoins, des désirs et des pulsions de chacun. Sans même évoquer le risque d'un basculement dans le règne généralisé de la loi de la jungle ou du développement, sur la base de la haine de soi, de nouvelles formes de régimes mortifères et totalitaires faisant suite aux précédents du $\mathrm{XX}^{\mathrm{e}}$ siècle, la conséquence du développement de cet individualisme, que les logiques et les intérêts capitalistes ont de leur côté évidemment intérêt à soutenir et favoriser, pourrait être l'instauration d'un rapport foncièrement consumériste à l'Ecole, voire la demande de son abolition pure et simple ou de sa réduction à un service d'accompagnement et de coaching individuels, venant dans tous les cas menacer foncièrement le projet même — et le désir — des générations adultes de protéger les jeunes générations par le moyen de l'éducation et de la formation.

$2^{\circ}$ ) En ne s'étonnant pas qu'aux yeux de beaucoup, il n'y ait pas d'autre option pour prévenir cette évolution que de défendre les anciennes configurations d'assujettissement, en tentant ou bien de les restaurer, ou bien d'en proposer de nouvelles versions ainsi que le fait par exemple M. Gauchet lorsqu'il propose de refonder l'Ecole sur le développement du sens de la «prééminence » et de la « transcendance » du monde historique et culturel ${ }^{2}$, on soutiendra à leur encontre, d'une part que de telles entreprises supposent toujours de ne pas avoir pris la mesure de la profondeur de cette crise dans laquelle nous sommes entrés, et d'autre part qu'elles continuent de se prévaloir d'évidences morales et rationnelles qu'elles tiennent pour fondatrices alors que cette crise atteint les configurations mêmes qui sont porteuses de ces évidences et dans lesquelles elles font sens. Ces entreprises ne peuvent par suite nullement garantir ni l'efficacité ni la pertinence même de l'alternative qu'elles proposent.

$3^{\circ}$ ) On soutiendra cependant qu'il existe encore pour l'Ecole au moins une autre possibilité, à la mesure de la crise actuelle et de sa profondeur, d'évoluer et de se transformer ou plutôt de véritablement muter et se reconfigurer en réorientant l'ensemble de son entreprise éducatrice et formatrice. Et on soutiendra que cette possibilité n'est nullement hors de portée ni ne relève a fortiori de l'idéalisation et de l'utopie, puisqu'elle va même dans le sens de certaines des préconisations de l'institution scolaire comme de certaines pratiques pédagogiques qu'on trouve déjà mises en œuvre par certains enseignants, mais de façon seulement locale et ponctuelle et qui ne peut qu'entrer en tension et contradiction vives avec le régime dominant et persistant de l'assujettissement.

A quoi tiendrait cette mutation? On pourrait dire qu'elle serait liée au projet inédit de chercher à "sur-individualiser les individus" en travaillant à les former comme des sujets actifs, critiques, créatifs et profonds. Là où les configurations d'assujettissement visaient à ce que les sujets ne développent leurs compétences réflexives, critiques et rationnelles que pour pouvoir être mis en situation d'autoassignation et d'auto-inhibition protectrices, il s'agirait désormais de constituer des sujets engagés dans un travail permanent d'extension et d'approfondissement de toutes leurs expériences. Corrélativement, il s'agirait qu'au lieu de faire table rase de toutes les expériences, de tous les pré-savoirs, de toutes les représentations et de tous les affects des élèves (rejetés du côté du domaine inessentiel du " privé » et du règne illusoire de «l'opinion »), l'Ecole prenne au contraire précisément pour point de départ ces expériences, ces pré-savoirs, ces représentations et ces affects en laissant advenir les récits et les verbalisations que les élèves peuvent en faire, puis les

${ }^{2}$ Cf. M.-C. Blais, M. Gauchet et D. Ottavi, Conditions de l'éducation, Paris, Stock, 2008, IIIème partie, « Fin ou métamorphose de l'autorité ? », p. 152, p. 155, p. 169 et passim, p. 160-171. 
réactions, les commentaires, les associations d'idées, les interrogations... des autres élèves, et en suscitant sur cette base un travail en commun d'élaboration, d'analyse, de réflexion, de questionnement des expériences, mais aussi de "perlaboration » au sens freudien des affects qui leur sont éventuellement associés.

L'enseignant lui-même n'interviendrait plus dans ce travail - et il ne ferait plus sens qu'il y intervienne - de manière frontale-directive, mais, avec la double habileté du gouverneur rousseauiste des premiers livres de l'Émile, il ferait toujours en sorte d'être perçu par les élèves comme un participant pleinement engagé en même temps qu'eux et avec eux dans les élaborations en cours, tout en sachant introduire discrètement et opportunément les éléments pouvant les conduire à découvrir progressivement de nouveaux objets, aspects et questions. Dans cette nouvelle configuration, le passé et l'histoire ne seraient plus par ailleurs présentés et prescrits comme extériorité purement factuelle ou comme héritage constituant, mais seraient rencontrés et découverts comme ce qui agit et laisse des traces profondes au cœur même du présent et demande à ce titre à y être mis à jour et déchiffré ; ils génèreraient en même temps de nouvelles expériences donnant à éprouver la contingence et l'être en devenir du présent ou à mesurer l'altérité des formes de vie et des représentations du monde même peu éloignées de nous. De même, l'art cesserait d'y être appréhendé soit comme domaine d'agrément et de loisir, séparé du travail, soit comme réalisation supérieure de l'humanité et patrimoine " fondateur », pour être reconnu comme champ ouvert, et aux frontières a priori inassignables, enveloppant toutes les expériences singulières de création, rencontrées elles-mêmes à partir des expériences immanentes que les élèves seraient amenés à faire, au cœur même du travail d'élaboration-perlaboration, de la création individuelle et collective de nouvelles expressions, de nouvelles compréhensions et de nouvelles interrogations. Et il n'y aurait sans doute plus de place non plus pour les injonctions de responsabilité et les prescriptions de devoirs de l'éducation morale et civique, là où l'expérience directe du réel social et politique engagerait les élèves à regarder en face et élaborer-perlaborer les phénomènes de conflits et de violences, tandis que leur expérience même du mode coopératif de travail et de vie de la classe les engagerait spontanément et "naturellement» à des pratiques de recherche de bien commun, de jugement de conflits, d'institution de règles... qui ne seraient plus d'ordre moral et républicain, mais éthique et démocratique.

Cette nouvelle configuration d'École, où l'on reconnaît sans doute des éléments caractéristiques de certaines pédagogies "actives", notamment coopératives et institutionnelles, mais qui s'en distingue également notamment par la place centrale donnée à la perlaboration et à la création, cette nouvelle configuration constitueraitelle une rupture décisive avec le régime multi-séculaire des configurations d'assujettissement ? C'est ce que l'on soutiendra, en mettant en avant qu'elle consisterait bien en la mise en œuvre d'un nouveau processus de subjectivation conduisant à la formation d'un nouveau type de sujets, répétons-le, actifs, critiques, créatifs et profonds. Il faut souligner ce qualificatif inhabituel et que presque personne n'ose employer aujourd'hui: des sujets profonds. Pourquoi profonds? Parce qu'au lieu d'être assignés, et de manière qui ne peut rester fondamentalement que partielle et finalement superficielle, à des Lois supérieures, les sujets se trouveraient au contraire constitués comme des sujets pleinement engagés et mis aux prises avec l'ensemble du réel, c'est-à-dire avec l'ensemble de ce qu'ils peuvent rencontrer, percevoir, éprouver... au travers de toutes leurs expériences, et disposés non seulement à s'en saisir, à l'analyser et à y réfléchir de façon critique, mais à en faire constamment un objet et un matériau de création, mobilisant donc toutes leurs ressources et leurs potentiels. Et telle est aussi la raison pour laquelle on peut penser que cette nouvelle configuration, qui apparaîtrait 
également porteuse d'un nouveau processus d' «émancipation », pourrait être de nature, si elle était mise en œuvre de façon conséquente et assumée, à susciter à nouveau une ample adhésion et même un ample et inédit désir collectif d'École, contrant accessoirement du même coup la menace de basculement dans le régime de l'individualisme hédoniste.

Douée d'un tel potentiel de restructuration et pouvant sembler si proche de nous voire déjà partiellement à l'œuvre dans certaines pratiques, cette mutation est-elle sur le point d'advenir de façon globale en saisissant l'ensemble du "système" scolaire et sociétal ? Force est de reconnaître qu'il y a des conditions - notamment sociales et politiques - pour qu'une société puisse vouloir entrer dans un tel projet d'éducation et de formation des sujets, et que ces conditions non seulement n'apparaissent pas réunies aujourd'hui, mais semblent peut-être encore plus compromises qu'il y a quelques décennies du fait des évolutions en cours, notamment techniques et économiques, et de leurs effets croissants de superficialisation et de pulsionalisation des intériorités psychiques. 